\title{
Single loop-and-clips technique (KING closure) for gastrotomy closure after transgastric ovariectomy: a survival experiment
}

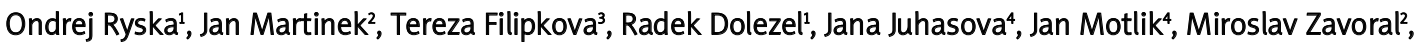 \\ Miroslav Ryska ${ }^{1}$ \\ 1Department of Surgery, $2^{\text {nd }}$ Medical Faculty, Charles University and Central Military Hospital, Prague, Czech Republic \\ 2Department of Internal Medicine, $1^{\text {st }}$ Medical Faculty, Charles University and Central Military Hospital, Prague, Czech Republic \\ ${ }^{3}$ Department of Surgery, Domazlice Hospital, Czech Republic \\ ${ }^{4}$ Institute of Animal Physiology and Genetics, Academy of Sciences, Libechov, Czech Republic
}

Videosurgery Miniinv 2012; 7 (4): 233-239 DOI: 10.5114/wiitm.2011.28870

\begin{abstract}
Introduction: A safe closure technique of transluminal access is essential for the widespread application of natural orifice transluminal endoscopic surgery (NOTES).

Aim: To evaluate the feasibility and effectiveness of a novel single loop-and-clips closure technique (KING closure). Material and methods: An experimental survival study using female laboratory pigs was performed. A gastrotomy was performed using a standard percutaneous endoscopic gastrostomy technique. A peritoneoscopy with an ovariectomy was then performed with a double-channel endoscope, on a total of 14 pigs. Two different techniques of gastrotomy closure were analysed: a loop-and-clips closure technique $(n=7)$ and a standard closure using endoclips $(n=7)$. After a follow-up period of 30 days, the animals were euthanized for post-mortem examination.

Results: In the "loop-and-clip" closure group, the correct placement of an endoloop and clips was achieved in all animals. At necropsy, no animal showed signs of an abscess or peritonitis. Histological examination demonstrated a patent full-thickness gastric wall closure without evidence of local complications in all instances. In the "clips" group, the gastrotomy closure was assessed as probably unsafe in three animals. At necropsy 3 (42.9\%) abscesses and $1(14.3 \%)$ case of peritonitis were found.

Conclusions: A single loop-and-clips closure technique (KING closure) represents a feasible, simple and effective method of gastric incision closure. It appears to be superior to the standard endoscopic closure technique using clips.
\end{abstract}

Key words: natural orifice transluminal endoscopic surgery, gastric closure, clips, endoloop.

\section{Introduction}

Natural orifice transluminal endoscopic surgery (NOTES) is a new evolving technique. The placement of surgical and endoscopic instruments is performed through a natural orifice [1]. Safe closure of the access site is a prerequisite for NOTES to be imple- mented in clinical practice. There are several closure techniques being tested in experimental settings today. These techniques can be divided into two major groups: the first group is characterized by the use of commercially available endoscopic accessories (clips, endoloops, stents, etc.) [2-4]. The second group incorporates several newly developed devices and 
prototypes especially designed for NOTES purposes (T-tags, Eagle-claw, staplers, etc.) [5-8]. Due to the uncertain efficacy and safety of using these methods via the stomach or the rectum, the majority of human procedures have used vaginal access with a standard hand-sutured closure [9]. Currently, there is no widely accepted closure device. Therefore, a simple and effective closure technique using commonly available endoscopic devices may still have its place in NOTES procedures. In addition to NOTES applications, the aforementioned techniques may also be used for the closure of other defects such as perforations or fistulas.

\section{Aim}

The aim of our study was to assess the feasibility, efficiency, and safety of the novel single loop-andclips gastric closure technique (KING closure) and compare it to a closure technique using endoscopic clips in pigs undergoing a pure NOTES intra-abdominal intervention (NOTES ovariectomy).

\section{Material and methods}

In the beginning, a non-survival experiment employing a gastrotomy with a subsequent KING closure technique was performed under laparoscopic control in one female pig. This pilot training study showed that the loop-and-clips closure technique provided a full-thickness, serosa-to-serosa closure (Figure 1).

Later, 14 female domestic mini pigs underwent NOTES peritoneoscopy with an ovariectomy in a nonrandomized survival study. Animals weighing $20 \mathrm{~kg}$

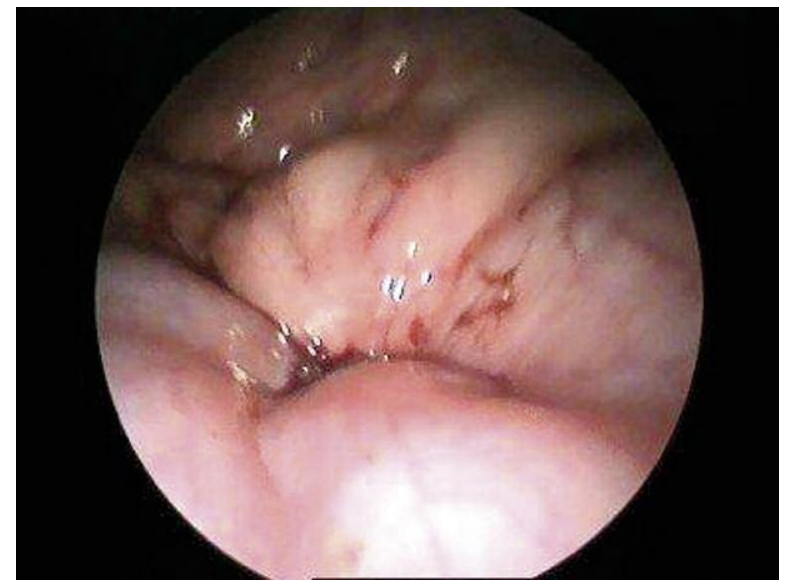

Figure 1. KING closure - laparoscopic control (range 11.6-31.6 kg) were fed with a liquid diet for two days before the procedure and then fasted overnight. Premedication with ketamine $10 \mathrm{mg} / \mathrm{kg}$ (Narkamon 1\%, SPOFA, Czech Rep.) and atropine $0.2 \mathrm{mg}$ (Atropin Biotica 0.5 mg, BB Pharma, Czech Rep.) was given intramuscularly $30 \mathrm{~min}$ before the procedure. Oral intubation was performed, a peripheral ear vein cannula placed, and maintenance anaesthesia given using $1.5 \%$ isoflurane and fentanyl $(3-5 \mathrm{ml} / \mathrm{h})$. Antibiotics were administered neither before nor after the procedure.

The protocol was approved by the Committee for the Protection of Animals of the Czech Academy of Sciences and the experiment was performed in accordance with Act no. 246/1992.

\section{Natural orifice transluminal endoscopic surgery procedure}

All procedures were performed using a doublechannel endoscope (CF 2T160l; Olympus Optical Co, Tokyo, Japan). An air flow inside the endoscope was used for insufflation. A percutaneous endoscopic gastrostomy (PEG) technique was used to gain access into the peritoneal cavity. A gastric wall incision measuring $15-20 \mathrm{~mm}$ was then performed with a tightened sphincterotome (KD-V411M-0330 Papillotome, Olympus Medical Co.) and the endoscope was advanced into the peritoneal cavity. No balloon was used for dilation of the access site.

The right ovary was exposed and then resected using both standard biopsy forceps and a snare with a electrocoagulation. The endoscope together with the resected ovary was then drawn back into the stomach and the peritoneal cavity desufflated. After the ovary was withdrawn through the mouth, the endoscope was reintroduced for a gastric wall closure.

In the first group of seven animals, a gastric wall closure was performed using the novel closure technique with one endoloop and 4-5 clips (Figure 2). An open endoloop with a $30 \mathrm{~mm}$ diameter (HX-400V-30 Aset PolyLoop Colo5, Olympus Medical System Co, Tokyo, Japan) was first fixed around the gastrotomy site using 4-5 clips (EZ clips, HX-610-135, Olympus medical (Co). The clipping device was then withdrawn from the right channel, and a large endoscopic grasper (F6-48L-1EMR, Olympus Medical System Co, Tokyo, Japan) was advanced via an open endoloop. Consequently, both edges of the incision were 


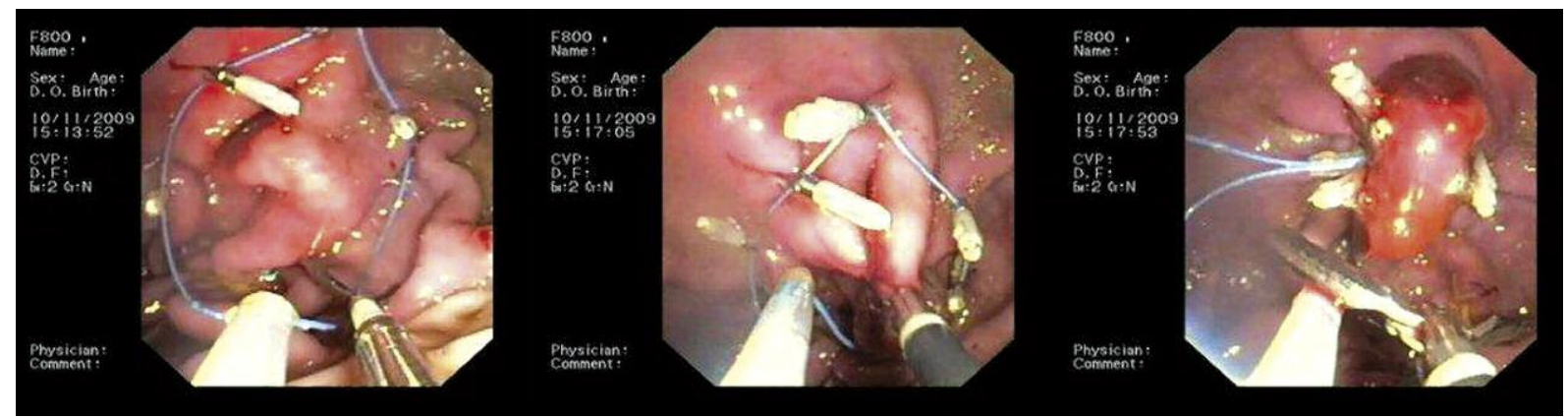

Figure 2. A novel single loop-and-clips closure (KING closure) technique

clasped with the grasper and pulled through the endoloop toward the endoscope. The endoloop was then closed and released. A small polyp-like formation was created at the gastrotomy site.

In the second group, also with seven animals, gastric wall incisions were closed using clips (EZ clips, HX610-135, Olympus Medical Co). An endoscopic grasper was used for tissue approximation in animals with difficult clipping, i.e. due to a large incision or the outside orientation of the incision margins. The clips were placed either starting from the middle, or starting at the edges and continuing towards the centre of the incision, depending on the current situation.

\section{Postoperative period}

All pigs recovered well after extubation and were placed in an animal facility unit where they were monitored twice a day for signs of any complications, feeding behaviour, and general well-being. All pigs resumed pig chow on the first postoperative day. Survival was assessed on the $30^{\text {th }}$ postoperative day, at which point the animals were euthanized and a necropsy performed. Tissue samples of the gastric wall closure site were obtained for histological examination in all seven animals only in the "loop-andclips" group.

\section{Statistical analysis}

For the purpose of descriptive statistics, data are presented as mean values with standard deviation. Nonparametric (Wilcoxon test) or parametric tests (Student's $t$-test) including Fisher exact test (frequency of complications) were performed to compare the groups. Statistical significance was defined as a $p$ value of less than 0.05 .

\section{Results}

\section{Survival experiment}

The main results are shown in Table I. Access into the peritoneal cavity was gained within 3-7 min in all animals. A consecutive peritoneoscopy did not show

Table I. Comparison between two observed groups (KING loop-and-clips technique vs. standard clips technique) concerning perioperative and necropsy parameters

\begin{tabular}{|lccc|}
\hline Parameter & KING closure $(n=7)$ & Clips $(n=7)$ & Value of $p$ \\
\hline Weight $[\mathrm{kg}]$ & $20.3 \pm 4.4$ & $23 \pm 8.5$ & NS \\
\hline No. of clips used & $5(3-6)$ & $5(2-5)$ & NS \\
\hline Procedure time [min] & $30 \pm 14$ & $45 \pm 17$ & 0.0425 \\
\hline Closure time [min] & $12 \pm 5$ & $21 \pm 6$ & 0.0201 \\
\hline Clinical complications, $n(\%)$ & $0(0 \%)$ & $0(0 \%)$ & NS \\
\hline Complications at necropsy (abcess and/or peritonitis), $n(\%)$ & $0(0 \%)$ & $4(57 \%)$ & 0.049 \\
\hline Adhesions, $n(\%)$ & $3(43 \%)$ & $3(43 \%)$ & NS \\
\hline
\end{tabular}

Unless specified otherwise, data are presented as mean values \pm standard deviation, NS - not significant 
any damage to adjacent organs. All ovariectomies were performed without major technical difficulties.

\section{Closure safety}

All gastrotomy sites were successfully closed using the loop-and-clips closure technique. An endoscopic view of a complete closure was achieved in all cases. A full distention of the stomach using air insufflation was obtained in all animals at the end of the procedure. In the "clip" group, three closures were assessed as probably unsafe based on their endoscopic appearance. However, a full distention of the stomach using air insufflation was also achieved in all cases. The loop-and-clips closure technique was faster (12 $\pm 5 \mathrm{~min}$ ) compared to the clipping technique $(21 \pm 6 \mathrm{~min} ; p<0.05)$. The number of clips required for closure was similar in both groups (five clips per animal on average).

\section{Necropsy findings}

The complication rate was higher in the "clips" group as compared with the "loop-and-clips" group. In the "clips" group, an intra-abdominal abscess was found in 3 cases, and signs of mild peritonitis were present in one animal (Table I). Small adhesions between the stomach and omentum were observed in 3 cases in each group.

All sutures in both groups healed completely and were airtight 30 days after the procedure (Figures 3 and 4). Clips or loops were not found in a single case. Histological findings showed a full-thickness closure healing of the gastric wall with a fibrotic scar in the muscular layer and flat mucosa (Figure 5).

\section{Discussion}

Safe closure technique is still the main issue in NOTES research, and has been defined as one of the major priorities by many authorities $[10,11]$. The ideal closure method should be easy to use, safe and effective. It should comply with current surgical standards to provide a serosa-to-serosa full thickness closure. Endoloop-based techniques (with or without clips) have been demonstrated to provide a safe and efficient closure of gastrotomy sites. The "QUEENS closure" was the first endoloop-based technique. It uses two endoloops of differing size and several clips [12]. However, the QUEENS closure is too complicated and time consuming. For instance, one endoloop has to be fixed alongside the endoscope and left open around the access site during the entire NOTES procedure. This is relatively easy for a simple diagnostic peritoneoscopy [13], but can prove rather difficult for a more complex and long lasting NOTES procedure.

Hucl et al. described a modified endoloop-based technique for the closure of a NOTES gastric wall incision. The closure was performed using an endoscopic grasper and subsequent application of two endo-
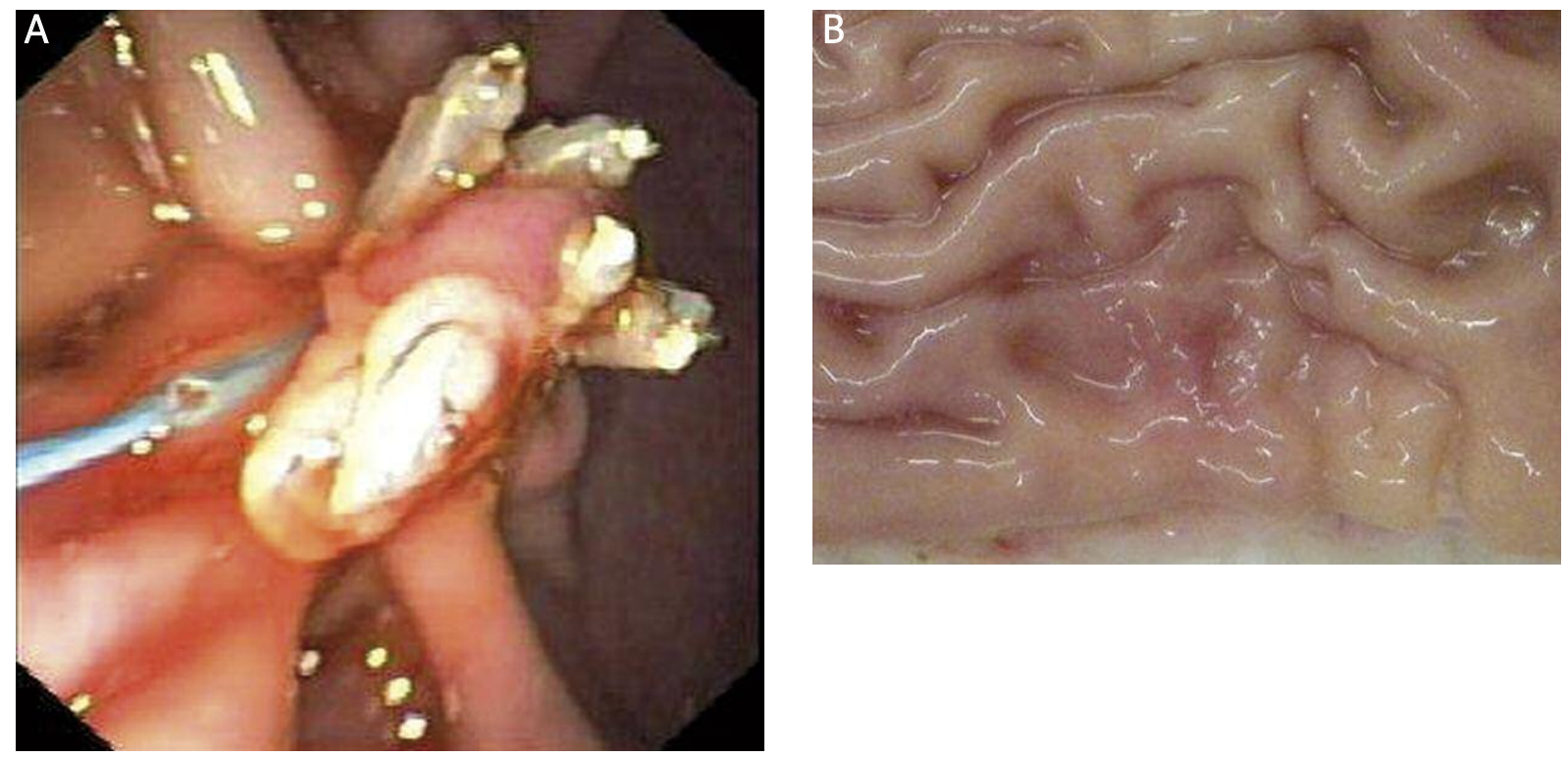

Figure 3. Gastrotomy closure using single loop-and-clip technique - endoscopic view (A) and necropsy view (B) 

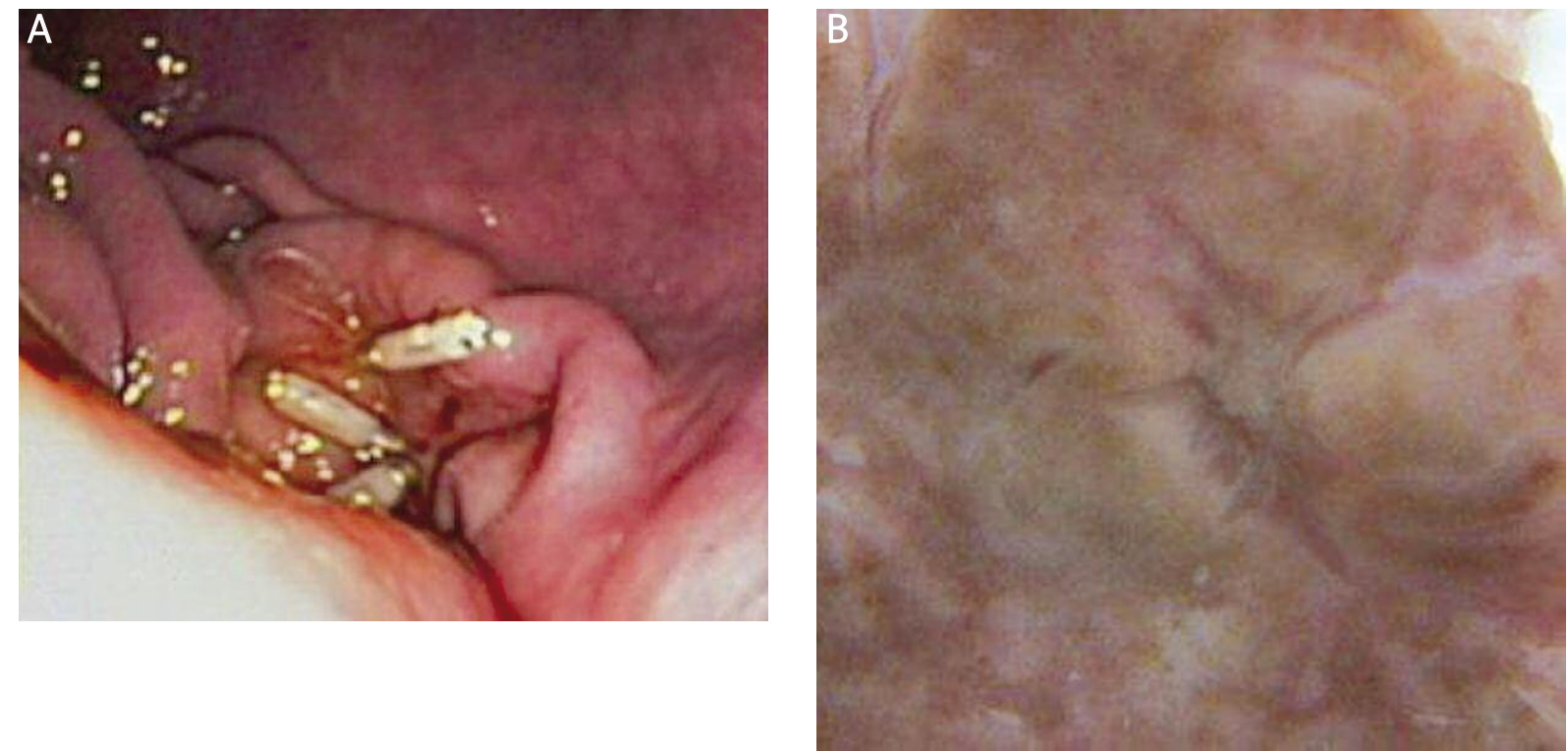

Figure 4. Gastrotomy closure using standard endoclips - endoscopic view (A) and necropsy view (B)

loops without clips [6]. This procedure seems to be easy to perform and safe and provides a full-thickness closure.

In the current study, we tested a novel endoloopbased technique for the closure of NOTES gastrotomy sites using a large endoscopic grasper, 3-5 clips and one endoloop. The technique was quick, with a median closure time of $12 \mathrm{~min}$. The loop-and-clips technique was faster even though a similar number of clips was used in both groups. The reason was that applying one simple clip correctly (closing both margins of the incision) was technically more challenging compared with clipping the opened endoloop.

The pilot non-survival experiment under laparoscopic control together with histological examination demonstrated a full thickness closure approximating all layers of the gastric wall. Importantly, the "KING" animals did not develop serious intra-abdominal complications in comparison to animals treated with a closure using endoscopic clips. In our study, the complication rate in the "clip" group was slightly higher as compared to previous studies [14]. We think this can be explained by the use of a double-channel endoscope, leading to a larger access site, which is more difficult to close completely by using simple clips. It is likely that such therapeutic endoscopes or platforms will be used in the future for sophisticated intra-abdominal procedures.

Our study was unique in that it tested a novel closure technique in a real NOTES intervention (ovariecto-

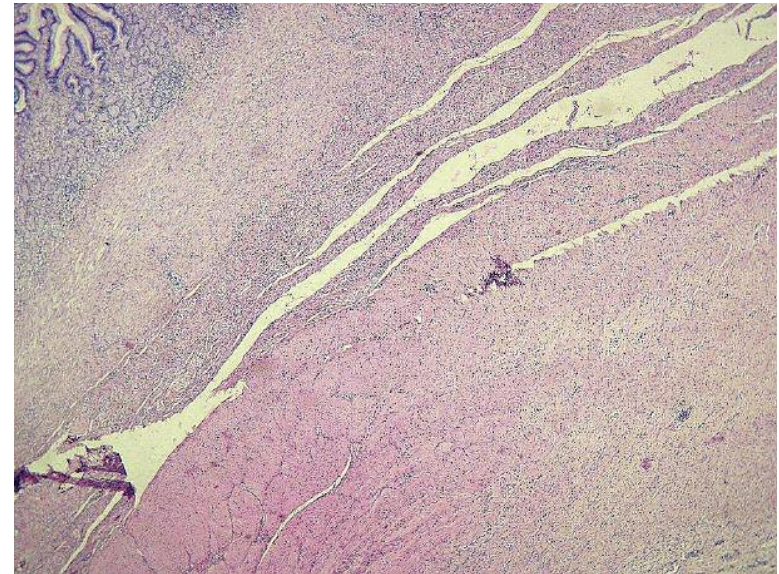

Figure 5. Histological findings of a KING closure 30 days after the procedure

my) and not in a simple peritoneoscopy as has been the case in earlier endoloop-based studies $[6,11]$.

A study comparing new over-the-scope (OVESCO) clips with standard clips also showed the superiority of the OVESCO clips. In this study, NOTES gastrotomy closure using standard endoclips was associated with an increased risk of leakage and intra-abdominal infections compared to the over-the-scope clips [15]. Those results are similar to our study, and can be explained by the insufficient patency of the closure technique using clips, especially when a large double channel endoscope is used. Standard clips provide a mucosal layer closure only; this is not sufficient for 
the closure of a large access site after a NOTES procedure [16].

On the other hand, the major problem that has been noted with endoloops is their tendency to slip over the gastrotomy site on release. We did not experience such difficulties in any instance, perhaps due to the fixation of the endoloop around the incision with clips. In our opinion, the most important step for a successful application of our method is the efficient grasping of both incision margins. The stomach of a pig is less elastic compared with humans, and its wall is thick. These conditions make the grasping manoeuvre more difficult. Nevertheless, it was successful in all animals. Once both margins were well and fully grasped, the application of an endoloop was very easy.

There is no study available that directly compares different endoloop-based techniques. Therefore, it is not known which of the available methods is superior. A study comparing these methods should be performed. Also, a study comparing the new over-thescope clip with an endoloop-based technique would be beneficial. Assuming similar effectiveness of both approaches, the economic advantage of an endoloopbased technique may be considerable.

The endoloop-based technique may also be applied to the closure of iatrogenic defects encountered inadvertently during an endoscopy (perforation) or for the closure of different kinds of fistula orifices. These methods may prove superior to the current practice, where such defects are closed with endoclips. Furthermore, before a widely accepted NOTES closure device becomes available, an endoloop-based technique may be used effectively in experimental NOTES studies.

There are several limitations to our study. It is a feasibility study only and the sample size is small; also, the study was not randomized. We did not perform validated leak tests, and the stomach tissue in pigs treated with the standard clips closure technique was not examined histologically. Finally, since an animal model was used, its application to human medicine must remain in question.

\section{Conclusions}

We tested a novel single-loop and clips closure technique (KING closure) and proved it to be safe, easy to perform, and effective. This new method seems superior to the standard closure technique using standard endoclips.

\section{Acknowledgments}

The authors would like to thank Dr. PO. Park (Department of Surgery, University Hospital - Östra, Gothenburg, Sweden) and Dr. D. Wilhelm (Department of Surgery, Klinikum r. d. Isar, Technical University, Munich, Germany) for their active scientific support. The authors would also like to thank Daniela Kaminova and Hana Rihova for their technical assistance. Finally, the authors thank Olympus Czech Republic for providing technical support.

Supported by a grant from IGA- NS9994-4 and by the Academy of Sciences of the Czech Republic (institutional research plan AV0Z50450515).

\section{References}

1. Swain P. A justification for NOTES-natural orifice translumenal endosurgery. Gastrointest Endosc 2007; 65: 514-6.

2. Kalloo AN, Singh VK, Jagannath SB, et al. Flexible transgastric peritoneoscopy: a novel approach to diagnostic and therapeutic interventions in the peritoneal cavity. Gastrointest Endosc 2004; 60: 114-7.

3. Ryou M, Fong DG, Pai RD, et al. Transluminal closure for NOTES: an ex vivo study comparing leak pressures of various gastrotomy and colotomy closure modalities. Endoscopy 2008; 40: 432-6.

4. Hookey LC, Bielawska B, Samis A, et al. A reliable and safe gastrotomy closure technique assessed in a porcine survival model pilot study: success of the Queen's closure. Endoscopy 2009; 41: 493-7.

5. Sclabas GM, Swain P, Swanstrom LL. Endoluminal methods for gastrotomy closure in natural orifice transenteric surgery (NOTES). Surg Innov 2006; 13: 23-30.

6. Hucl T, Benes M, Kocik M, et al. A novel double-endoloop technique for natural orifice transluminal endoscopic surgery gastric access site closure. Gastrointest Endosc 2010; 71: 806-11.

7. Chiu PW, Lau JY, Ng EK, et al. Closure of a gastrotomy after transgastric tubal ligation by using the Eagle Claw VII: a survival experiment in a porcine model (with video). Gastrointest Endosc 2008; 68: 554-9

8. Voermans RP, Worm AM, van Berge Henegouwen MI, et al. In vitro comparison and evaluation of seven gastric closure modalities for natural orifice transluminal endoscopic surgery (NOTES). Endoscopy 2008; 40: 595-601.

9. Kobiela J, Stefaniak T, Dobrowolski S, et al. Transvaginal NOTES cholecystectomy in my partner? No way! Videosurgery Miniinv 2011; 6: 236-41.

10. Inui K. Natural orifice transluminal endoscopic surgery: a step toward clinical implementation? Gastrointest Endosc 2007; 65: 694-5. 
11. Meining A, Feussner H, Swain P, et al. Natural-orifice transluminal endoscopic surgery (NOTES) in Europe: summary of endoscopy. 2011; 43: 140-3.

12. Hookey LC, Khokhotva V, Bielawska B, et al. The Queen's closure: a novel technique for closure of endoscopic gastrotomy for natural-orifice transluminal endoscopic surgery. Endoscopy 2009; 41: 149-53.

13. Michalik M, Bobowicz M, Trybull A, et al. Diagnostic pure transgastric NOTES in an intensive therapy unit patient. Videosurgery Miniinv 2011; 6: 108-10.

14. Wagh MS, Merrifield BF, Thompson CC. Survival studies after endoscopic transgastric oophorectomy and tubectomy in a porcine model. Gastrointest Endosc 2006; 63: 473-8.

15. von Renteln D, Vassiliou MC, Rothstein RI. Randomized controlled trial comparing endoscopic clips and over-the-scope clips for closure of natural orifice transluminal endoscopic surgery gastrotomies. Endoscopy 2009; 41: 1056-61.

16. Arezzo A, Morino E. Endoscopic closure of gastric access in perspective NOTES: an update on techniques and technologies. Surg Endosc 2010; 24: 298-303.

Received: 8.03.2012, Revised: 29.03.2012, Accepted: 23.04.2012. 